IOSR Journal of Pharmacy

ISSN: 2250-3013, www.iosrphr.org

||| Volume 2 Issue 4 || || July-August 2012 ||| PP.04-16

\title{
The Strengths, Weaknesses, Opportunities and Threats (SWOT) Analysis of HIV Type-1
}

\author{
Babalola, Michael Oluyemi. \\ Department of Microbiology, Adekunle Ajasin University, P.M.B. 001, Akungba Akoko, Ondo State, Nigeria.
}

\begin{abstract}
I hereby bring into perspective, the Strengths, Weaknesses, Opportunities and Threats (SWOT) analysis of HIV Type -1 and the first proposal for the application of this innovative concept in the field of HIV research, to holistically devise strategies at undermining the prowess of the Human Immunodeficiency Virus and overcoming its scourge. The strengths of HIV are the biological attributes that facilitate the pathogenicity, establishment of infection and spread of the virus. They include, the possession of Reverse Transcriptase enzyme for reverse transcription of RNA genome to DNA, ability to maintain persistence by forming a provirus, ability to specifically target and replicate in the immune cells, antigenic mimicry and adoption of host derived glycan shield for immune evasion, HIV-1 strains harbor enhanced biological fitness upon recombination, HIV has exceptionally high rate of replication and disrupts the Blood-Brain Barrier.

The weaknesses of HIV are the internal attributes and requirements for survival of the virus that invariably present as opportunities and targets for elimination. These include: the requirement for CCR5 and CXCR4 chemokine co-receptors for fusion prior to internalization, presence on HIV-1 envelope of conserved determinants that mediate CD4 binding, the Achilles heel of HIV is a tiny stretch of amino acids numbered 421 to 423 on gp 120, the capsid proteins reveal potential weakness at the inner core of the virus, HIV proteins retain virus internal carbohydrate, and the virus thrives on human proteins.

The various host factors that were harnessed as opportunities for infection by HIV include, the ability to establish infection via multiple portals of entry, Sexually Transmitted Infections(STIs) increase the susceptibility to HIV-1 infection, STIs boost HIV shedding in the genital tract and amplifies infectiousness, presence of hidden HIV reservoirs that serve as foci of dissemination, the genital anatomical structure of the female genital tract aids biological susceptibility, the immune status of the host, and underlying illness or subsequent exposure to a bandwagon of opportunistic pathogens.

The Threats to HIV are the external conditions that are inimical to the virus establishment and which may be harnessed for elimination of the pathogen. They include: limited host reservoir, the virus is susceptible to a Lectin isolated from ripened banana fruits as potent inhibitor of HIV replication, the development and application of Abzymes, development of agents that can selectively target only HIV infected cells, sustenance of global AIDS funding, and evolution of a potent HIV / AIDS Vaccine.
\end{abstract}

\section{INTRODUCTION}

Acquired Immune Deficiency Syndrome (AIDS) is a specific group of diseases or conditions which are indicative of severe immunosuppression related to infection with the Human Immunodeficiency Virus (CDC, 1997). Globally, 33.3 million people live with the virus, of which 2.7 million children under 15 years are estimated to be infected with the virus and 2.6 million were newly infected in 2009 (WHO, 2010). Today, HIV is reputed to be the greatest health crisis the world faces. Since the start of the HIV epidemic 21 million people have died and 57 million people have become infected.

There are two main types of the virus; HIV-1 and HIV-2. Both types are transmitted by sexual contact, through blood and from mother to child, and they appear to cause clinically similar AIDS. It is now generally accepted that cross-species transmission to humans of SIVcpz in central Africa and SIVsm in West Africa gave rise to HIV-1 and HIV-2, respectively.

HIV-1 is prevalent worldwide. It consists of four subgroups designated M (Major), $\mathrm{N}$ (New), O (Outlier) and P. These four groups may represent four separate introductions of Simian immunodeficiency virus into humans. Group O and group N- an extremely rare strain that was discovered in Cameroon in 1998 appear to be restricted to West- central Africa. In 2009, a new strain closely relating to gorilla Simian Immunodeficiency Virus was discovered in a Cameroonian woman. It was designated HIV-1 group P (Plantier, 2009). However, more than $90 \%$ of HIV-1 infections belong to group M. 


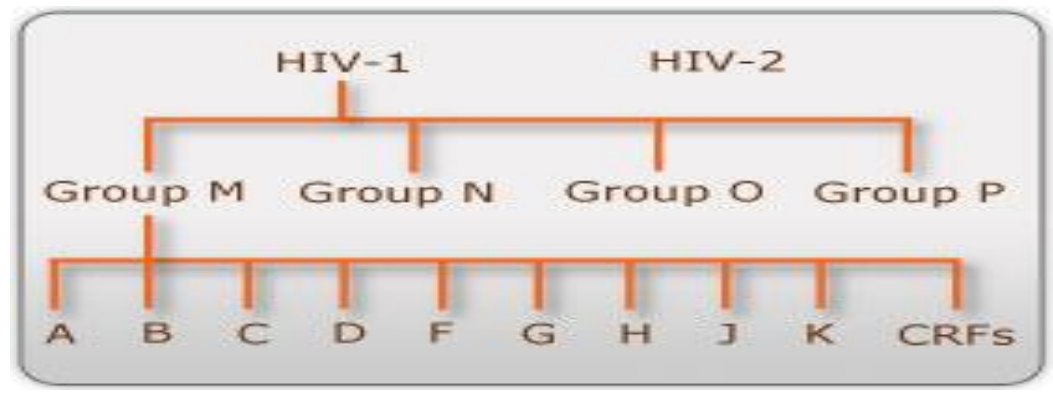

Fig 1: The different levels of HIV classification (Courtesy Avert.org)

The M subgroup has nine (9) genetically distinct subtypes namely A, B,C, D, F, G, H, J, K and CRFs. Occasionally, two viruses of different subtypes can meet in the cell of an infected person and recombine their genetic material to create a new hybrid virus ( a process similar to sexual reproduction, and sometimes called "viral sex"). Many of these new strains do not survive for long, but those that infect more than one person are known as circulating recombinant forms or CRFs. For example, the CRF A/B is a mixture of subtypes A and B. The HIV-1 subtypes and CRFs are very unevenly distributed throughout the world, with the most widespread being subtypes A and C. Subtype A and CRF A/G predominate in West and Central Africa, with subtype A possibly also causing much of the Russian epidemic ( Bobkov, et al., 2004). Historically, subtype B has been the most common subtype / CRF in Europe, the Americas, Japan and Australia. Although this remain the case, other subtypes are becoming more frequent and now account for at least $25 \%$ of new HIV infections in Europe. Subtype C is predominant in Southern and East Africa, India and Nepal. It has caused the world's worst HIV epidemics and is responsible for around half of all infections. Subtype D is generally limited to East and Central Africa. CRF A/E is prevalent in South-East Asia, but originated in Central Africa. Subtype F has been found in Central Africa, South America and Eastern Europe. Subtype G and CRF A/G have been observed in West and East Africa as well as Central Europe. Subtype H has only been found in Central Africa; J only in Central America; and K only in the Democratic Republic of Congo and Cameroon.

HIV-2 is only prevalent in West Africa, it is less easily transmitted, the period between initial infection and illness is longer, and it consists of six subtypes, namely; A, B, C, D, E and F.

Infections with lentiviruses typically show a chronic course of disease, a long period of clinical latency, persistent viral replication and involvement of the central nervous system. Using electron microscopy, HIV-1 and HIV-2 resemble each other strikingly. However, they differ with regard to the molecular weight of their proteins, as well as having differences in their accessory genes. HIV-2 is genetically more closely related to the SIV found in sootey mangabeys (SIVsm) rather than HIV-1 and it is likely that it was introduced into the human population by monkeys. Both HIV-1 and HIV-2 replicate in CD4+ T-cells and are regarded as pathogenic in infected persons, although the actual immune deficiency may be less severe in HIV-2 infected individuals.

HIV is a diploid, positive sense, single stranded RNA Virus belonging to the family Retroviridae. HIV1 viral particles have a diameter of $100 \mathrm{~nm}$ and are surrounded by a lipoprotein membrane. Each viral particle contains 72 glycoprotein complexes, which are integrated into this lipid membrane, and are each composed of trimers of an external glycoprotein gp120 and a transmembrane spanning protein gp41. The bonding between gp120 and gp41 is only loose and therefore gp120 may be shed spontaneously within the local environment. Glycoprotein gp120 may also be detected in the serum as well as within the lymphatic tissue of HIV-infected patients. During the process of budding, the virus may also incorporate different host proteins from the membrane of the host cell into its lipoprotein layer, such as HLA class I and II proteins, or adhesion proteins such as Intracellular Adhesion Molecule-1 (ICAM-1) that may facilitate adhesion to other target cells. The matrix protein p17 is anchored to the inside of the viral lipoprotein membrane. The p24 core antigen contains two copies of HIV-1 RNA. The HIV-1 RNA is part of a protein-nucleic acid complex, which is composed of the nucleoprotein $\mathrm{p} 7$ and the reverse transcriptase p66 (RT). The viral particle contains all the enzymatic equipment that is necessary for replication: a Reverse Transcriptase (RT), an Integrase p32 and a Protease p11 enzymes (Fig. 2). 


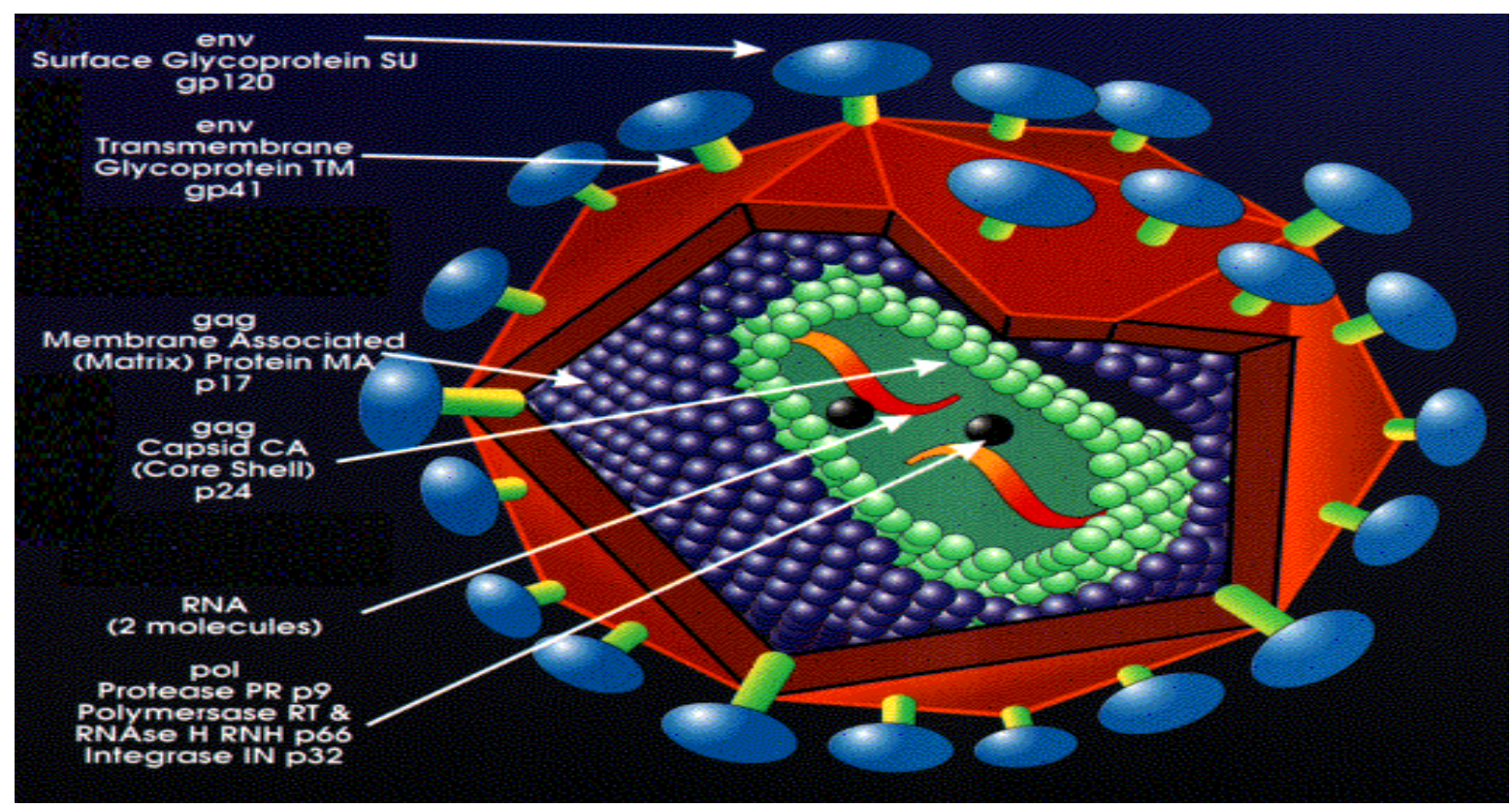

Fig. 2: HIV-1 Particle

The objective of this presentation is to bring into perspective the characteristic Strengths, the Weaknesses, the Opportunities and Threats to HIV-1 that will enable scientists to consider and devise multifactorial strategies to holistically overcome the scourge of HIV -1 infections.

\section{THE CONCEPT OF SWOT}

SWOT is an acronym for Strengths, Weaknesses, Opportunities, and Threats . It was originally designed by the Boston Consulting Group (BCG) in Massachusetts, USA, where Strategic Managers devised the strategy to undermine their competitors and achieved competitive advantage.

SWOT analysis can be adapted to scientific research to helps us as Scientists in formulating strategies that augment our research and enable us to understand and devise holistic strategies to combat emerging and recalcitrant infectious pathogens. When the characteristic capacities and the gaps have been identified, we can then devise means of achieving our goals.

\section{THE STRENGTHS OF HIV -1}

The Strengths of HIV are the biological attributes that facilitate the pathogenicity, establishment of infection and spread of the virus. These include:

The Possession of Reverse Transcriptase enzyme which enhances genetic flexibility and production of viral variants. The lack of proof reading ability of reverse transcriptase leads to synthesis of error-prone viral DNA during reverse transcription ( $3 \tilde{\mathrm{A}}-10-5$ mutations/nucleotide/replication cycle). This, in addition to the high recombination frequencies accompanying reverse transcription are responsible in part to the heterogeneity of the virus.

Ability to maintain persistence by integration of the viral genome in the cellular chromosome of the host forming a provirus, especially in resting memory CD4 T-lymphocyte cells, and build up a lifelong infection.

Ability to specifically target and replicate in the immune cells. The human immune system is composed of specialized cells and organs that work together to find and kill invaders. It is responsible for distinguishing the foreign proteins of invading pathogens and for protecting against alien substances and infections. It can reject transplanted organs, but it is also susceptible to pathogen attacks, from HIV in particular. The main cellular targets for HIV-1 are the CD4+ T-helper subset of lymphocytes, CD4+ cells of the macrophage lineage, and some populations of dendritic cells (DC). These are sets of immune cells that prompts the cytotoxic T- cells as well as the B-cells to initiate actions against the pathogen.

Antigenic mimicry: HIV adopts host derived glycan shield for immune evasion. Most of the antigenic surface of HIV is covered by carbohydrates, It's often called the glycan shield. The virus needs to be continually evading the immune system and this shield is perhaps its most effective strategy (Dunlop et al., 2010).

Possession of auxiliary genes (e.g. Viral infectivity factor, Vif and Nef) that facilitate intercellular spread and immunogenicity. NEF is a multifunctional 27-kd myristoylated protein and one of the first HIV proteins to be produced in infected cells. It is the most immunogenic of the accessory proteins. The nef genes of 
HIV and SIV are dispensable in vitro, but are essential for efficient viral spread and disease progression in vivo. Nef is necessary for the maintenance of high viral loads and for the development of AIDS in macaques, and viruses with defective Nef have been detected in some HIV-1 infected long term survivors. Nef downregulates $\mathrm{CD} 4$, the primary viral receptor, and MHC class I molecules, and these functions map to different parts of the protein. It increases viral infectivity (NIH, 2010).

(i) REV. The second necessary regulatory factor for HIV expression. A 19-kD phosphoprotein, localized primarily in the nucleolus/nucleus. Rev is considered the most functionally conserved regulatory protein of Lentiviruses. Rev cycles rapidly between the nucleus and the cytoplasm.

(ii) VIF. Viral infectivity factor, a basic protein typically $23 \mathrm{kD}$. It promotes the infectivity but not the production of viral particles. In the absence of Vif, the produced viral particles are defective, while the cell-tocell transmission of virus is not affected significantly. In 2003, it was discovered that Vif prevents the action of the cellular APOBEC-3G protein, which deaminates DNA:RNA heteroduplexes in the cytoplasm. , Vif induces the degradation of APOBEC $3 \mathrm{G}$, thereby counteracting its antiviral activity.

(iii) VPU Vpu (viral protein U) is unique to HIV-1, SIVcpz (the closest SIV relative of HIV-1), SIV-GSN, SIVMUS, SIV-MON and SIV-DEN. There is no similar gene in HIV-2, SIV-SMM, or other SIVs. Vpu is a 16-kd (81-amino acid) type I integral membrane protein with at least two different biological functions: (a) degradation of CD4 in the endoplasmic reticulum, and (b) enhancement of virion release from the plasma membrane of HIV-1-infected cells. Env and Vpu are expressed from a bicistronic mRNA. Vpu probably possesses an N-terminal hydrophobic membrane anchor and a hydrophilic moiety. It is phosphorylated by casein kinase II at positions Ser52 and Ser56. Vpu is involved in Env maturation and is not found in the virion. Vpu has been found to increase susceptibility of HIV-1 infected cells to Fas killing (NIH, 2010).

HIV -1 strains harbor enhanced biological fitness upon recombination. In Malaysia where Circulating Recombinant Forms (CRFs) predominate, co-circulation of CRF01-AE and subtype B has resulted in the emergence of CRF33-01B with accompanied higher apoptotic cell death and syncytial induction compared to subtype B (Katherine et al., 2009). Biological and molecular comparisons of CRF33_01B against its parental subtypes clearly show that CRF33_01B replicated better in activated whole peripheral blood mononuclear cells (PBMCs) and CD4+ T-lymphocytes, but not monocyte-derived macrophages (MDMs). Also, its acquired fitness was greater than CRF01_AE but not subtype B. These adaptive and survival abilities could have been acquired by CRF33_01B due to the incorporation of subtype B fragments into the gag-RT region of its full-length genome (Lau et al., 2010).

The Human Immunodeficiency Virus is an RNA virus with inherent capacity to vary, recombine and diversify. It is a highly variable virus which mutates very readily. This means there are many different strains of HIV, even within the body of a single infected person. This gives it a clear edge to evade the human immune system, to continuously replicate and survive in the host and mount resistance against some antiviral drugs. No two HIV-1 isolates were identical. When subjected to nucleotide sequence analysis, even HIV-1 samples recovered from a single individual exhibited significant heterology. Some of the nucleotide changes resulted in amino acid substitutions (nonsynonymous) whereas others did not alter the protein sequence (synonymous). Although nucleotide changes were distributed throughout the HIV-1 genome, the greatest variability occurred in the gene encoding the envelope (Env) glycoprotein, gp160, when intra- or interpatient virus specimens were compared. The term quasispecies was subsequently coined to describe the pool of diverse and changing populations of virus present in an individual infected with HIV-1. The genetic diversity of HIV-1 poses significant challenges for diagnosis and treatment of new HIV infections. Optimal drug treatment requires ongoing, precise measurement of viral levels. If variant subtypes are present and significantly underquantified or undetected, therapy could be compromised.

The genetic variability of the Human Immunodeficiency Virus is associated with disease progression and death. Previous reports stated that Ugandans infected with subtype D or recombinant strains incorporating subtype D developed AIDS sooner than those infected with subtype A, and also died sooner, if they did not receive antiretroviral treatment. subtype D was claimed to be more virulent because it is more effective at binding to immune cells (Laeyendecker et al., 2006). This was corroborated by another study in 2007, which found that Kenyan women infected with subtype D had more than double the risk of death over six years compared with those infected with subtype A ( Baetan et al., 2007). A study of sex workers in Senegal found that women infected with subtype C, D or G were more likely to develop AIDS within five years of infection than those infected with subtype A (Kanki et al., 1999). In addition, several studies conducted in Thailand suggest that people infected with CRF A/E progress faster to AIDS and death than those infected with subtype B, if they do not receive antiretroviral treatment( Nelson et al., 2007).

The inherent ability of the virus to continually change in a patient, portrays physicians, as chasing a moving target. When an antiviral is being developed against a known strain, it has actually changed within a short time thereby rendering the antiviral ineffective against the viral particle. In addition, neutralizing antibodies do exist in HIV-1-infected individuals, however, there is a time lag in their appearance. That is, 
individuals will develop neutralizing antibodies to their own viruses with time, however, by the time these antibodies develop, the new viruses circulating in the individual's plasma will become resistant to neutralization, even though the older ones are now sensitive to the current antibodies in the patient's serum. Thus, the antibody response appears to be hitting a 'moving' target, allowing viruses to escape continuously. Further knowledge gained on understanding the mechanisms of humoral escape will likely lead to potential new therapies.

HIV has exceptionally high rate of replication that is potentially capable of overwhelming the immune response of the host. The high levels of progeny virus production in vivo $\left(10^{9}\right.$ particles/day; 150 to 300 replication cycles/year) is enormous for the immune response of the host. During acute HIV-1 infection, the virus replicates extensively in the absence of any detectable adaptive immune response, reaching levels of over 100 million copies HIV-1 RNA/ml. It is during this initial cycle of viral replication that important pathogenic processes are thought to occur. These include the seeding of virus to a range of tissue reservoirs and the destruction of CD4+ T-lymphocytes, in particular within the lymphoid tissues of the gut. The very high levels of HIV-1 viremia are normally short-lived, indicating that the host is able to generate an immune response that controls viral replication. Over the following weeks, viremia declines by several orders of magnitude before reaching a viral setpoint. This setpoint, following resolution of the acute infection, is a strong predictor of longterm disease progression rates (Mellors, 1995).

HIV Distrupts the Blood- Brain Barrier. HIV has been found to cause neurological damages by weakening the blood- brain barrier- a network of blood vessels that keeps potentially harmful chemicals and toxins out of the brain- by overtaking a small group of supporting brain cells. The findings help explain why some people living with HIV experience neurological complications, despite the benefits of modern drug regimens that keep them living longer. Standard antiretroviral treatments successfully suppress the replication of HIV and slow the progression of the disease. Recent studies show that 40 to $60 \%$ of patients on ART continue to experience mild to moderate neurological deficits - including memory loss and learning challenges. HIV infection in a small number of supporting brain cells called astrocytes breaks down the blood- brain barrier, despite low to undetectable viral production. Under normal conditions astrocytes help bolster the blood vessels comprising the barrier. The presence of HIV in about 5\% of astrocytes led to the death of nearby uninfected cells and made the barrier more permeable. As the neighboring cells died, however, HIV infected astrocytes survived. This study provides a possible explanation indicating that minute numbers of infected astrocytes can trigger a cascade of signals that could open the brain to various toxic influences. Astrocytes exchange chemical signals through specialized molecules called gap junctions. New therapies are needed that not only target the virus, but also to stop the virus from spreading damage to other uninfected brain cells (Eugenin et al., 2011) .

HIV-1 induced killing is the predominant cause of CD4+ T-cell death and is caused by a necrotic, not an apoptotic, mechanism. The principal hallmark of apoptosis, the activation of cysteinyl aspartaterequiring proteases (known as caspases), is typically not detected during virus infection of cultured human $\mathrm{T}$ cell lines exhibiting extensive cytopathic effects.

No specifically identified immunogens that elicit broadly neutralizing antibodies. This phenomenon has hampered the efforts of researchers seeking to develop a truly prophylactic HIV Vaccine. Many neutralizing antibodies either do not or only poorly show inhibition of primary viral isolates. Amajor problem lies in the high variability of the gp120 glycoprotein itself. Furthermore, gp120 epitopes may be highly glycosylated and certain structural domains are hidden, at least temporarily, so that immunodominant epitopes may not be recognized (Chen, 2005).

\section{THE WEAKNESSES OF HIV -1.}

The weaknesses of HIV are the internal attributes and requirements for survival of the virus that invariably present as opportunities and targets for elimination. These include:

The requirement for chemokine co-receptors CCR5 and CXCR4 for fusion, prior to internalization of the virus. HIV-1 isolates using the CXCR4 receptor (now designated X4 viruses) are usually TCL-tropic, Syncytial inducing (SI) strains that exhibit enhanced cytopathicity and more vigorous replicative properties. In contrast, HIV-1 strains exclusively using the CCR5 receptor (R5 viruses) are predominantly M-tropic and Non syncytial inducing ( NSI). Dual-tropic R5/X4 strains exhibiting a continuum of tropic phenotypes are frequently SI.

The HIV -1 envelope retains conserved determinants that mediate CD4 binding. The envelope surface is involved in the meta-stable attachment of CD4 before the external glycoprotein gp120 rearrangement that is required for stable engagement (Tongqing et al., 2007). Gp120 primarily binds to certain epitopes of CD4. Binding to CD4 induces conformational changes in gp120 that promote a more efficient interaction of the V3 loop of gp120 with its respective coreceptor. Membrane fusion is dependent on gp120 coreceptor binding. Gp41, as the transmembrane part of the envelope glycoprotein gp160, is crucial for the fusion of the viral and 
the host cell membrane. Similar to influenza hemagglutinin, it was postulated that consequent to the binding of gp120 to CD4, a conformational change is induced in gp41 that allows gp41 to insert its hydrophobic NH2 terminal into the target cell membrane. Unlike the changeable regions of its envelope, HIV needs at least one region that must remain constant to attach to cells. If this region changes, HIV cannot infect cells. Equally important, HIV does not want this constant region to provoke the body's defence system. So, HIV uses the same constant cellular attachment site to silence B lymphocytes - the antibody producing cells. The result is that the body is fooled into making abundant antibodies to the changeable regions of HIV but not to its cellular attachment site. Immunologists call such regions superantigens. HIV's cleverness is unmatched. No other virus uses this trick to evade the body's defences.

The Achilles heel of HIV is a tiny stretch of amino acids numbered $421-433$ on gp120. This is a constant region that does not change if the virus must remain infectious. The weak spot is hidden in the HIV envelope protein gp120. This protein is essential for HIV attachment to host cells, which initiate infection and eventually lead to Acquired Immunodeficiency Syndrome or AIDS. Non lethal mutations of the region can be effected to render the virus non infectious.

HIV Capsid proteins reveal potential weakness at the inner core of the virus. This is a novel target to distrupt capsid assembly which may prevent the formation of infectious particles (Mark and Barbie, 2007).

HIV Proteins retain virus internal carbohydrate that enables the immune system to recognise virus specific signatures. The exceptionally dense packing of the carbohydrates on HIV's coat turns out to be another possible Achilles' Heel of the virus. The carbohydrates on HIV's proteins are so densely packed that there is no room for the trimming and modification that would normally take place as the protein completes its journey out of the cell. HIV's proteins therefore retain their immature 'internal' carbohydrates - a carbohydrate array made up of mannose sugar residues. This presents an opportunity for the immune system to 'see' a virus-specific signature on the cell. And unlike the constantly changing viral proteins, this carbohydrate structure is relatively fixed "Self-sugars" in the wrong place, internal- type sugars that would never normally be seen on the surface. They are available to the immune system for recognition (Dunlop et al., 2010)

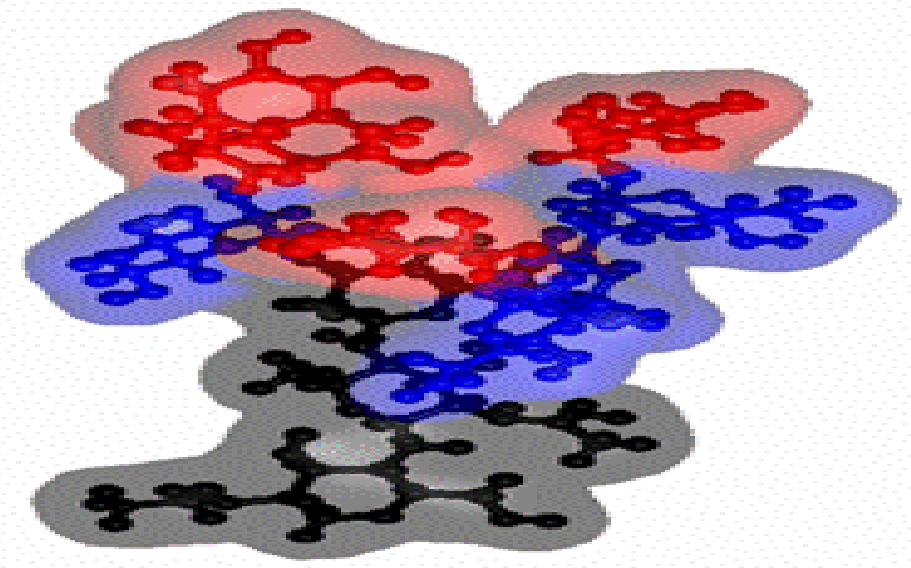

Fig. 3: One of HIV's sugars showing the tips of the branches (red) which the immune system can recognize and which happen to be similar to sugar structures found on yeast polysaccharides (Dunlop et al., 2010)

HIV thrives on human host protein. Researchers have identified 273 human proteins that HIV needs to thrive. This landmark discovery opens new opportunities for scientists to fight the virus using therapeutic targets. Since HIV makes only 15 of its own proteins, it must commandeer human cell proteins for successful infection. A key advantage to using these host proteins as therapeutic targets is that the proteins are essential for functions that HIV cannot manage on its own. These host proteins are active in HIV's life cycle, helping it gain access to the cell, to integrate its genome into the cell's genome, replicate and leave the cell to infect other cells. Of the 273 proteins identified, only 36 were previously known to investigators. The newly pinpointed proteins could help researchers devise better treatment strategies to get around a major HIV treatment issue: the virus mutating to develop resistance to antiviral drugs. Many of the host proteins identified are more frequent in immune cells than other types of human cells. This helps explain why HIV is so difficult to treat—it takes over 
the very immune cells that the body needs to fight infection. The disadvantage of targeting these proteins, of course, is that blocking human proteins could prevent functions that are important for normal living and could result in serious side effects. However, there is precedent for this type of approach. Some cancer therapies work in a similar way by blocking the proteins that feed the fast-growing tumor cells without killing many other fastgrowing cells, such as those in the bone marrow. The techniques could be used to expose vulnerabilities in other viruses, such as hepatitis, which may rely on some of the same proteins identified (Brass et al., 2008).

Compulsory Integration of the viral genome to the host' is required for productive infection. A distinguishing feature of retrovirus replication is the insertion of a DNA copy of the viral genome into the host cell chromosome after reverse transcription. The integrated viral DNA (the provirus) serves as the template for the synthesis of viral RNA and is maintained as part of the host cell genome for the lifetime of the infected cell. Retroviral mutants deficient in the ability to integrate generally fail to establish a productive infection.

\section{OPPORTUNITIES OF HIV -1}

The Opportunities of HIV are the various host factors which were harnessed as advantages for infection. These include:

Ability to establish infection via multiple portals of entry. The inherent ability of the virus to survive in body fluids such as blood and blood products, semen, vaginal fluids, breastmilk, organ and tissue transplants, present opportunities for transmission via blood transfusions, scarification marks, sexual intercourse and breastfeeding.

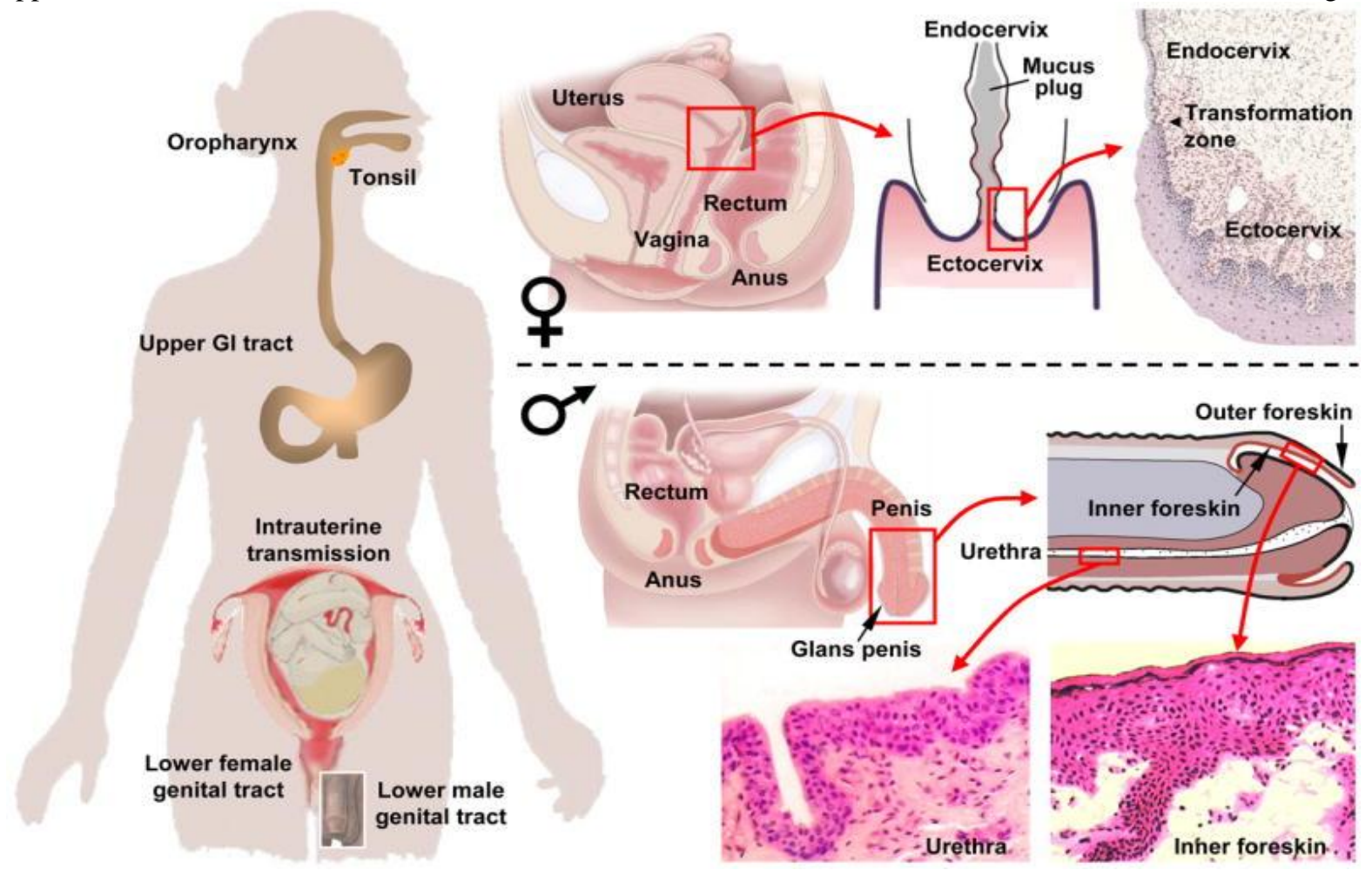

Fig.4: HIV Invasion Sites (Hladik and McElrath, 2008).

Most cases of paediatric HIV results from Mother to Child Transmission which contributes increasingly to childhood morbidity and mortality. Majority of infected children acquire the infection during pregnancy (in utero), during delivery (intrapartum) or during breastfeeding ( postpartum)(Fig. 4).

Sexually Transmitted Infections (STIs ) increase the susceptibility to HIV-1 infections by recruiting HIV susceptible inflammatory cells to the genital tract as well as distrupting mucosal barriers (Fig.4) to infection. Infections such as Syphilis, gonorrhea, Chancroid, Chlamydia and condylomata acuminata synergise HIV infection. STIs boost HIV shedding in the genital tract, amplifies infectiousness and susceptibility. The predominant mode of transmission of both HIV and other STIs is sexual intercourse. Strong evidence supports several biological mechanisms through which STIs facilitate HIV transmission by increasing infectiousness and HIV susceptibility. If the incidence/prevalence of STIs is high in a country, then there is the possibility of high rates of sexual transmission of HIV (UNAIDS/WHO, 2004). 
Presence of hidden HIV reservoirs. Current antiretroviral regimens are unable to totally eradicate the Human Immunodeficiency Virus in infected patients and this failure is attributable to HIV reservoirs. Somewhere inside the body, hidden HIV particles manage to escape drugs, and indefinitely provide new supplies of the virus. A region of the body was identified where HIV replication remains active, even when viral load in the plasma is undetectable after 10 years of infection, that is Long Term Non Progressors (Cumont et al., 2007). Higher levels of virus replication was found in the lymph nodes of the intestine's mesentery, compared to other regions. These levels were 20 to 30 times higher than what they were in other organs, and the immune system CD8+T cells responsible for killing HIV-infected cells-die prematurely in the mesenteric region.

Findings in molecular genetics suggests other mechanisms are also at play in this reservoir process. Some virus particles seem to escape medication by hiding inside cells of the immune system itself-in resting memory CD4+ T cells, also known as T-helper cells, that carry an integrated provirus that is transcriptionally silent. T-helper cells are present in all compartments of the body. They play a central role in the immune response by signaling other cells of the system to perform their functions. But during HIV infection, their cellular machinery is hijacked by the virus which multiplies and releases viral particles in the blood circulation. However, in a very few CD4 cells, the virus 'decides' not to replicate and remains in a latent state, The quasiabsence of viral gene expression enables the virus to remain 'invisible' and escape antiretrovirals-which mainly target replication. Some reactivation process may occur, and replication can start over, thus providing new supplies of the virus ( Wallace, 2009 ). In addition, HIV-1 replication in monocyte or macrophage lineage microglia within the CNS may persist in patients receiving highly active antiretroviral therapy (HAART) because of inefficient transfer of drugs across the blood-brain barrier. This viral reservoir could contribute to the rebound of plasma viremia invariably observed after cessation of drug administration.

Acquisition of multiple sexual partners and all forms of sexual aberrations that humans practice, such as homosexuality, sodomy, bisexuality, and so on.

Injecting drug use is a leading cause of the spread of HIV. In some parts of the world, heroin use and HIV have reached epidemic proportions

The genital anatomical receptacle or structure of the female genital tract aids biological susceptibility. Women are exposed to infectious fluids for longer periods during sexual intercourse than men are; they also face increased risk of tissue injury during intercourse. The cells lining the surface of the cervix may also be especially susceptible to HIV infection, and recent data suggest that vaginal cells are more susceptible than previously thought. Young women may be at particular risk because of ectopy, a condition in which the columnar epithelium (cells lining the transition zone of the cervical os) extends onto the face of the cervix. Cervical ectopy has been associated with increased risk of HIV infection, possibly because it facilitates greater exposure of these target cells to trauma and pathogens in the vagina (Higgins et al., 2010). Women of all ages are biologically and socially more vulnerable to HIV because of rape and forced sex. Forced sex is strongly linked with vaginal or anal tears that can expedite disease transmission, and abused women are less able to negotiate condom use (Randolph et al., 2007).

Immune status of the host. Irrespective of the levels of immune responses that the host is able to mount, HIV was still able to surmount such responses.

An underlying illness or subsequent exposure to a bandwagon opportunistic pathogens such as bacteria, viruses, fungi and protozoan that thrive on opportunities provided by the deficient immune status of the host. HIV / AIDS and Tuberculosis have caused an unprecedented global health crisis, accounting for more than 4 million deaths each year, with the majority of these in developing countries, particularly sub- Saharan Africa. Mycobacterium tuberculosis affects one third of the world's population and represents the main cause of death in HIV- infected patients. TB represents a dramatic problem in eastern Europe because it is highly drug resistantThis epidemiological situation is a major problem for all of Europe due to the impact of the spread of clinically relevant strains ( WHO, 2002).

Viral ability to penetrate some special sites. HIV in the brain and in the cerebro-spinal fluid may be particularly resistant to chemotherapy because of the failure of anti-retroviral drugs to penetrate the blood-brain barrier.

Presence of a "diagnostic window period" . On infection, it takes several weeks before antibodies become detectable. This phenomenon is called "diagnostic window" and is determined by the time period the body requires to produce detectable levels of antibodies (Busch, 1997). The switch from antibody-negative to antibody-positive is called "seroconversion". The screening tests currently used are able to recognize an HIV 
infection six weeks after primary infection in about $80 \%$ and after the 12th week in almost $100 \%$ of cases; only in very rare cases is an infection recognized only after six months.

Treatment interruptions. Many treatment interruptions occur without the clinician's knowledge. Almost all patients who stop treatment experience a "rebound" in viral load within a few weeks, even patients in whom this has been undetectable for several years (Chun, 2000). Viral load is usually detectable again within 10-20 days, and its doubling time in the blood is around 1.6-2.0 days. The viral load in compartments such as the CNS, as well as the semen and vaginal fluids, changes in parallel to that in the plasma. The patients should therefore be informed about the higher risk of transmitting HIV. Frequently, an initial overshooting rebound is observed, and only after a few weeks does the viral load settle to its original, pre-treatment level (Hatano, 2000). The rebounding virus evidently does not originate from latent reservoirs; other cell populations must exist, from which these new viruses can be produced so quickly ( Imamichi, 2001). Treatment interruptions can have serious immunological consequences. The loss of CD4 cells during an interruption may not be regained as quickly. Viral resistance always has to be anticipated whenever there is viral replication in the presence of suboptimal drug levels, and thereby resistant mutants gain a selective advantage over the wild-type virus. As a result, there are concerns that resistances could develop both during the washout phase of medication (increasing viral replication with insufficient plasma levels) and on re-initiation of treatment (continued replication despite sufficient plasma levels).

Side effects of Antiretroviral drugs. Patients on HAART commonly suffer from side effects. As a result, treatment of HIV infection has become a complicated balancing act between the benefits of durable HIV suppression and the risks of drug toxicity. About $25 \%$ of patients stop therapy within the first year on HAART because of side effects. About the same number of patients does not take the recommended dosages of their medication due to concerns regarding the side effects ( Chesney, 2000). Patients, who report significant side effects, are more often non-adherent to therapy (Ammassari, 2001). The patient should be counseled in detail about potential side effects such as gastrointestinal side effects, Nausea and vomiting, Diarrhea, Hepatotoxicity, Peripheral polyneuropathy, CNS disorders, Increased bleeding episodes, Allergic reactions, Avascular necrosis, and Osteopenia/osteoporosis (Carr and Cooper, 2001) among others. All of these provide leverage for the virus to rebound.

Poverty and malnutrition. The recalcitrant level of poverty and inadequate feeding and nutritious meals in developing countries aggravate susceptibility and risky social behaviours that facilitate contraction of the virus.

\section{THREATS TO HIV-1.}

The Threats to HIV are the external conditions that are inimical to the virus establishment and which may be harnessed for its elimination. These include:

Limited host reservoir. HIV is known to be pathogenic only in human as it has not been described to cross the species barrier or discovered to be a trans kingdom pathogen unlike some other viruses or fungi.

Improved rapid diagnostic procedures for prompt detection. The basis for effective treatment and cure of a patient is the rapid diagnosis of the disease and its causative agent, which is founded on the analysis of the clinical symptoms coupled with laboratory tests. As we approach the 21 st century, clinicians are becoming increasingly able to diagnose and treat diseases at the molecular level. The rapid development of new methods and techniques in the area of molecular biology has gained new insights into the genetic and structural features of a considerable number of human pathogens. Serodiagnosis by ELISA, Confirmation by Western blotting and specific detection by PCR( Udo, 1996) as well as the determination of the cellular and viral markers give clues to the combat and eradication of the Human immunodeficiency virus.

Scale up of a Lectin isolated from ripened banana fruits as potent inhibitor of HIV replication. Banlec is a jacalin-related lectin isolated from the fruits of bananas, Musa acuminata. This lectin binds to high mannose carbohydrate structures, including those found on viruses containing glycosylated envelope proteins such as the HIV Type -1 (Swanson et al., 2010). Although condoms are quite effective against the spread of HIV and some other sexually transmitted diseases, they are only effective if they are used consistently and correctly, which is often not the case (Randolph et al., 2007) This is particularly true in the developing world, where women have relatively little control over sexual encounters and, thus, have not been able to enforce condom usage, so the development of a long-lasting, self-applied, microbicide is very attractive. In fact, it is estimated that $20 \%$ coverage with a microbicide that is only $60 \%$ effective against HIV may prevent up to 2.5 million HIV infections over three years. Therefore, even modest success with microbicides could save millions of lives. Some of the most promising compounds for inhibiting vaginal or rectal HIV transmission are agents that block HIV before integration of the viral genome into the target cell. Thus, the viral entry step is one potential target for a microbicide. Lectins inhibit HIV-1 entry by binding to carbohydrate structures found on the viral envelope. Examples of anti-HIV lectins include Cyanovirin-N (CV-N), Griffithsin (GRFT), and snowdrop 
lectin (GNA). The HIV-1 envelope protein gp120 contains 20-30 possible N-linked glycosylation sites. These carbohydrate structures makeup 50\% of the molecular weight of the protein. Glycosylation affects aspects of the viral life cycle including protein folding, cellular transport, binding to cellular receptors, trans-infection by dendritic cells, and shielding from the immune response.

Male circumcision to prevent the spread of HIV . Circumcision of the male foreskin reduces the risk of infection from diverse sources through unprotected intercourse (Weiss, 2010) This is probably also true for HIV (Siegfried, 2006). In a large randomized study in Uganda on 4,966 men, the risk of infection with HIV decreased from 1.33 to $0.66 / 100$ person years (Gray, 2007). This effect is explained by the presence in the male foreskin (Fig.4) of CD4-positive Langerhans cells, which act as the primary target cell for the HIV infection. It has been estimated that in Africa alone circumcision could prevent approximately 2 million HIV infections in the next few years (Williams, 2006).

Development and application of Abzymes. Abzymes are endogenous retrovirus sequences that was discovered in HIV negative people with the autoimmune disease Systemic Lupus Erythematosus and a small number of HIV positive people who do not require treatment and do not get AIDS. Abzymes have the capacity to recognize and degrade all diverse forms of HIV (Yasushiro et al., 2008). "The abzymes recognise essentially all of the diverse HIV forms found across the world. This solves the problem of HIV changeability. Unlike regular antibodies, abzymes degrade the virus permanently. A single abzyme molecule inactivates thousands of virus particles. Regular antibodies inactivate only one virus particle, and their anti-viral HIV effect is weaker.

Prevention of HIV entry into cells. The virus surface glycoprotein gp41 and the cellular co-receptor CCR5 are drug targets to block entry (Lengauer et al. 2010).

Education, counseling and the use of condoms to disseminate knowledge on the virus and for prevention of further spread / transmission (WHO, 2010)

Availability and correct use of ART for prevention, treatment, reduction of viral load and infectiousness. These measures ensure the reduction and combating effectively the scourge.

Development of agents that can selectively target only HIV infected cells e.g Prostratin, an extract of Homolanthus nutan has been proven effective in flushing HIV reservoirs. The plant is used in traditional Western Samoan herbal medicine to treat infections, like hepatitis. The U.S. National Cancer Institutes found that prostratin has powerful anti-HIV activities. (ARA, 2009)

Barrier protection methods, topical microbicides and mucosal immunization . Microbicide is a substance that can reduce transmission of STDs (e.g., herpes simplex virus, HIV) when applied in the vagina or rectum. It could come in many forms, including gels, creams, suppositories, films, lubricants. The substance might protect against HIV in a variety of ways, such as killing HIV on contact, creating a physical barrier around vulnerable cells, or, strengthening the body's normal defenses.

Sustenance of Global AIDS funding, about 8 billion dollars (Tagline, 2009) to vigorously pursue the treatment and mitigating the impact of HIV-AIDS epidemic.

Evolution of a potent HIV/AIDS Vaccine. This remain the anticipated breakthrough. HIV and TB enter the human body at mucosal sites. Development of mucosally delivered vaccines against HIV and Mycobacterium tuberculosis, which will induce local immunity to neutralize the pathogens at their ports of entry and systemic immunity to prevent the systemic spread of the infection is the best mode. The right vaccine will be capable of inducing cell mediated and humoral immunity against the virus and virally infected cells in their different phases of the viral life cycle. Various developmental HIV/ AIDS vaccines are in progress. The EU Research strategies include, AIDS Vaccine Integrated Project, Recombinant Measles Virus as a Vector for HIV Vaccines, Development of a novel Therapeutic HIV-1 Vaccine: Horizontal Gene Transfer by Using Apoptotic HIV-1 DNA Containing Activated T-Cells, A Combined Pox -Virus / Lentiviral Vector System to Treat HIV infection: Immunization and Direct in vivo Gene Transfer in T- Lymphocytes., A Combined Microbicidal- Immunising Strategy Against SIV and HIV infection, Very Innovative AIDS Vaccine, Development of a New Vaccine against HIV: Virosomes Incorporating HIV Proteins, Explaining and Improving Efficacy of Targeted Immunodeficiency Virus-like Particle Vaccines against AIDS, Identification of Novel Epitopes as HIV-1 Vaccine candidates (EC, 2008).

Development of a virtual immune system known as the Immnunogrid to model the human immune system. The simulator presently works at the cellular level. Computer simulation will help understand the host immune response to attacks by pathogenic bacteria and viruses. The immunoGrid applications will provide tools for the clinicians and vaccine/ immunotherapy developers for identification of optimal immunization protocols. The unique component of the project is that it aims to connect molecular level interactions ( which regulate immune responses ) with system level models ( which study behavior of the immune system as a whole) - a novel approach to disease prevention and treatment.

Host genetic factors play important roles in both susceptibility and resistance to HIV-1 infection. The two known natural HIV-1 resistance states are the alloimmunity and homozygous CCR5 mutation. These is a deletion in the major coreceptor for entry of HIV-1 into CD4+ T-cells, a chemokine receptor called CCR5. 
Homozygotes for this 32 base pair deletion (CCR5delta32) do not express the receptor at the cell-surface and can only be infected with HIV strains that are able to use other coreceptors, such as CXCR4. Heterozygotes for the deletion exhibit significant lower viral setpoints and slower progression to AIDS. There is a striking resistance to HIV-1 infection in homozygous delta32 CCR5 mutation, found in about $1 \%$ of Caucasians. These individuals lack cell surface expression of CCR5 generate a large amount of CC chemokines ( CCL-3, CCL-4 and CCL-5) and may develop antibodies to CCR5, but they do not suffer from ill health. This can be adapted to potentiate resistance to the virus. In addition to mutations in the chemokine receptor genes, a number of HLA class I alleles have been described to be associated with both, lower viral setpoints and slower disease progression, including HLA-B27 and -B57 (O'Brien, 2001, ). Recent studies demonstrated that individuals expressing HLA-B57 presented significantly less frequently with symptomatic acute HIV-1 infection, mount strong virus-specific $\mathrm{T}$ cell responses restricted by these protective alleles, and exhibited a better control of viral replication following acute infection ( Altfeld, 2006).

Implementation of Highly Active Anti Retroviral Therapy (HAART). Currently, 25 single or combination preparations from four classes of drugs are licensed: nucleoside and nucleotide analogs (NRTIs) such as Lamivudine, Zidovudine Didanosine and Abacavir; non-nucleoside reverse transcriptase inhibitors (NNRTIs) such as Efavirenz and Nevirapine; protease inhibitors (PIs) such as Amprenavir, Indinavir, Saquinavir and Ritonavir; fusion inhibitors such as Enfuvirtide; and Entry inhibitors such as Selzentry ( Maraviroc).

\section{SWOT ANALYSIS}

Draw up a 2 by 2 contingency Table and fix in the identified characteristics. Taken together, for the Strengths and the Opportunities, all research and efforts should be directed at decreasing them while the focus should be at increasing all the characteristics that present as weaknesses of the virus and Threats to the virus.

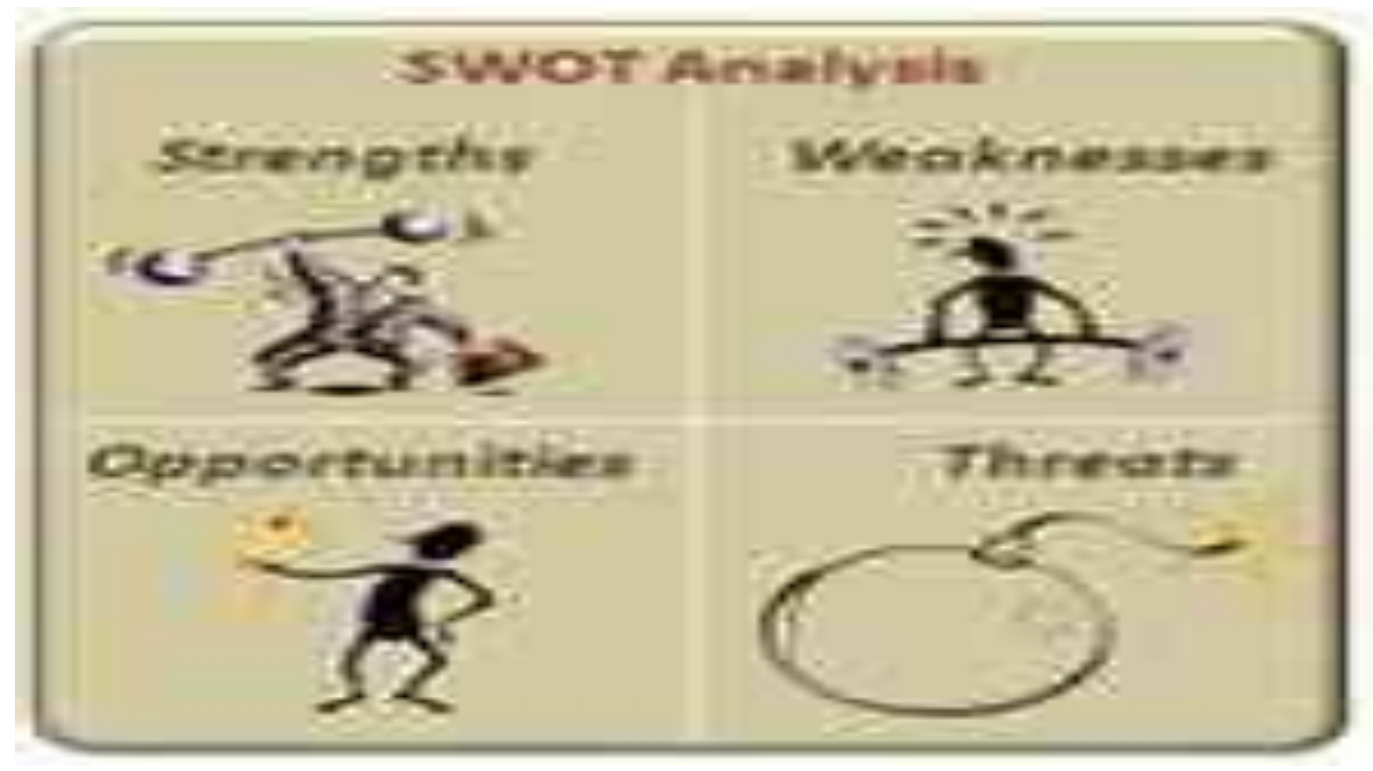

Fig 5: Animation of the Contingency Table of SWOT Analysis. 
Table 1: Contingency Table of the SWOT Analysis of HIV Type- 1.

DECREASE

\section{STRENGHTHS OF HIV-1}

-Possession of reverse transcriptase enzyme.

-Ability to maintain persistency.

-Ability to specifically target and replicate in the immune cells.

-Antigenic mimicry.

-Possession of auxiliary genes.

-Enhanced biological fitness upon recombination.

-RNA virus with inherent capacity to vary, recombine and diversify.

-Genetic variability is associated with disease progression and death.

-Exceptionally high rate of replication.

-HIV distrupts the blood- brain barrier.

-Necrotic induced killing of CD4+ T- cells.

-No specifically identified immunogens that elicit broadly neutralising antibodies.

\section{OPPORTUNITIES OF HIV-1}

-Ability to establish infection via multiple portals of entry.

-Most pediatric HIV cases results from Mother to Child transmission.

-STIs increase the susceptibility to HIV -1 infection. -STIs boost HIV shedding in the genital tract and amplifies infectiousness.

-Presence of hidden HIV reservoirs.

- Acquisition of multiple sexual partners and all forms of sexual aberrations.

-Injecting drug use.

-Genital anatomical structure of females aid biological susceptibility.

-Immune status of the host.

-Underlying illness or subsequent exposure to opportunistic pathogens.

-Treatment interruptions.

- Side effects of Antiretrovirals

- Poverty and Malnutrition

\section{INCREASE}

WEAKNESSES OF HIV-1

-Requirement for chemokine co-receptors, CCR5 and CXCR4.

for fusion prior to internalization.

-HIV-1 envelope retains conserved determinants that mediate CD4 binding.

- Achilles heel of HIV is a tiny stretch of amino acids numbered 421 to 423 on gp120.

-HIV capsid proteins reveal potential weakness at the inner core of the virus.

-HIV proteins retain virus internal carbohydrate.

-HIV thrives on human host protein.

-Compulsory integration of the viral genome to the host' genome

\section{THREATS TO HIV-1}

-Limited host reservoir.

-Improved rapid diagnostics for prompt detection.

-Use of isolated Lectins from ripened banana fruits as potent inhibitor of HIV replication.

-Male circumcision.

-Development and application of Abzymes that degrade all diverse forms of HIV.

-Prevention of HIV entry into cells.

-Education, counseling and the use of condoms.

-Availability and correct use of ART.

-Development of agents that can selectively target HIV infected cells e.g Prostratin from Homolanthus nutans.

-Sustenance of global HIV /AIDS funding.

-Evolution of a potent HIV/AIDS Vaccine.

-Host genetic factors in susceptibility and resistance

-Implementation of HAART

\section{IMPLICATIONS AND ADVANTAGES OF SWOT ANALYSIS \\ IV. DISCUSSION AND CONCLUSION}

All activities should be geared towards and focused on the design and implementation of research findings to drastically decrease or eliminate the Strengths and Oportunities of the virus e.g the evolution of drugs such as Efavirenz and Selzentri.

There is the need to conceptualize, design and implement new researches targeted at drastically weakening or increase the weaknesses of the virus and increasing threats to the virus.

We must understand that HIV explores its biological strengths, conserves its weaknesses and exploits the host opportunities, making it one of the most outstanding fittest surviving pathogen.

Innovative research is required in the discovery of the seemingly elusive magic bullets to eradicate HIV Pandemic. Scientists in the dicey field of HIV research must scale up all promising research findings and build more on the threats. The adoption of the SWOT analysis is an innovative research strategy that was hitherto not considered but highly promising to the discovery of solutions to combat HIV /AIDS.

By holistically harmonising all the identified factors, putting them into perspective and implementing our strategies, we are a step closer to winning the Nobel prize for overcoming HIV and its cohorts. 


\section{REFERENCES}

[1]. Aids Research Alliance (2009) Prostratin: A new approach Targeting HIV infected cells.

[2]. Altfeld, M., Kalife, E.T., Qi, Y., et al.,(2006). HLA Alleles Associated with Delayed Progression to AIDS Contribute Strongly to the final CD8(+) T Cell Response against HIV-1. PLoS Med. 3(10):403.

[3]. Baeten, D. et al, (2007)"HIV-1 subtype D infection is associated with faster disease progression than subtype A, in spite of similar HIV-1 plasma viral loads" 14th Conference on Retroviruses and Opportunistic Infections ( Abstract no. 68)

[4]. Bobkov, A.F., Kazennova, E.V., Selimova, L.M., et al, (2004)"Temporal trends in the HIV-1 epidemic in Russia: predominance of subtype A" J Med Virol.

[5]. Brass, A. L., Dykxhoorn, D. M., Benita, Y., et al.(2008) Identification of host proteins required for HIV infection through a functional genomic screen. Science 319: 921-6.

[6]. Busch, M.P. and Satten, G.A. (1997). Time course of Viremia and antibody seroconversion following human immunodeficiency virus exposure. Am. Journal Med. 102(suppl. 5B): $\quad$ 117-124.

[7]. Carr, A. and Cooper, D.A.(2001). Adverse effects of antiretroviral therapy. Lancet 356:1423-30.

[8]. Chen, B., Vogan, E.M., Gong, H., Skehel, J.J., Wiley, D.C. and Harrison, S.C.(2005). Structure of an unligated Simian Immunodeficiency Virus gp120 core. Nature 433:834- 841.

[9]. Chun, T.W., Davey, R.T. Jr., Ostrowski, M., et al., (2000).Relationship between pre-existing viral reservoirs and the reemergence of plasma viremia after discontinuation of HAART. Nat. Med. 6: 757-761.

[10]. Cumont, M. C. et al., (2007) "TGF-b in intestinal lymphoid organs contributes to the death of armed effector CD8 T cells and is associated with the absence of virus containment in rhesus macaques infected with the simian immunodeficiency virus," Cell Death Differ. 14 (10): 1747-1758.

[11]. Dunlop, L. M., Keith, R. I. , et al. (2010) Polysaccharide mimicry of the epitope of the broadly neutralizing anti - HIV antibody 2G12 induces enhanced antibody responses to self oligommannose glycans. Glycobiology 3 (12)

[12]. European Commission, EC (2008) Vaccines for human . Research funded by the European Union. Office for Official publication of the European Communities.

[13]. Gray, R., Kigozi, G., Serwadda, D., et al., (2007). Randomized trial of male circumcision for HIV prevention in Rakai, Uganda, Abstract 155LB, 14th CROI, Los Angeles. Abstract: http://www.retroconference.org/2007/Abstracts/30666.htm

[14]. Hatano, H., Vogel, S., Yoder, C., et al.,(2000). Pre- HAART HIV burden approximates post-HAART viral levels following interruptions of therapy in patients with sustained viral suppression. AIDS 14: 1357-1363.

[15]. Higgins, J.A., Hoffman, S. and Dworkin, S.L. (2010) Rethinking Gender, Heterosexual Men, and Women's Vulnerability to HIV/AIDS AJPH 100(3): 435-445

[16]. Hladik, F. and McElrath, M.J. (2008) Setting the stage - HIV host invasion Nature Rev. Immun. 8 (6):447-457.

[17]. Imamichi, H., Crandall, K.A., Natarajan, V. et al.,(2001). HIV Type-1 quasi-species that rebound after discontinuation of HAART are similar to the viral quasi species present before initiation of therapy. J. Infect. Dis. 183: 36-50.

[18]. Kanki, P.J., Hamel, D.J., Sankale, J-L et al.(1999) "Human Immunodeficiency Virus Type 1 Subtypes Differ in Disease Progression" Journal of Infectious Diseases 179 (1)

[19]. Katherine, L., Wang, B., Miranda-Saksena, M., et al. (2009) Emerging trends of HIV epidemiology in Asia. AIDS Review 9 (4): $218-219$.

[20]. Laeyendecker, O., Li. X., Arroyo, M., et al.(2006) "The Effect of HIV Subtype on Rapid Disease Progression in Rakai, Uganda" 13th Conference on Retroviruses and Opportunistic Infections (abstract no. 44LB)

[21]. Lau, K.A., Wang, B., Miranda-Saksena, M., Boadle, R., et al.,.(2010) Evidence for possible biological advantages of the newly emerging HIV-1 circulating recombinant form from Malaysia - CRF33_01B in comparison to its progenitors - CRF01_AE and subtype B. Curr. HIV Res. 8 (3): 259-271.

[22]. Mark, Y. and Barbie, G. (2007) Structure of HIV Capsid protein reveals potential weakness at inner core of the virus. Cell 68 (3): $89-91$

[23]. Mellors, J.W., Kingsley, L.A., Rinaldo, C.R. et al., (1995). Quantitation of HIV-1 RNA in plasma predicts outcome after seroconversion. Annals of internal Med.122: 573-579.

[24]. Nelson, K. E., et al. (2007) "Survival of blood donors and their spouses with HIV-1 subtype E (CRF01 A E) infection in Northern Thailand, 1992-2007", AIDS 21 (Supplement 6).

[25]. NIH (2010) Landmarks of the HIV genome. HIV sequence database.

[26]. O'Brien, S.J., Gao, X. and Carrington, M. (2001). HLA and AIDS: a cautionary tale. Trends Mol. Med. 7: $379-381$.

[27]. Plantier, J.C. (2009) 'A new human immunodeficiency virus derived from gorillas', Nature Medicine

[28]. Randolph, M. E., Pinkerton, S. D., Bogart, L. M., Cecil, H., and Abramson, P.R. (2007) Sexual pleasure and condom use Arch. Sex. Behav. $36(6): 844-848$

[29]. Siegfried, N., Muller, M., Deeks, J., et al.(2005) HIV and male circumcision--a systematic review with assessment of the quality of studies. Lancet Infect Dis 5: 165-73.

[30]. Swanson, M. D., Winter, H. C., Goldstein, I . J., and Markovitz, D .M.( 2010). A Lectin Isolated from Bananas Is a Potent Inhibitor of HIV Replication. The journal of biological chemistry 285 ( 12): 8646-8655.

[31]. Thomas, L., Andre, A., et al . (2010) Chasing the AIDS Virus. Communications of the ACM 53 (9): $66-74$.

[32]. Tongqing, Z., et al. (2007) Structural definitions of a conserved neutralization epitope on HIV - 1 gp120. Nature 445: 732 737.

[33]. Udo Reischl (1996). Application of molecular biology-based methods to the diagnosis of infectious diseases. Frontiers in Bioscience 1: 72-77.

[34]. UNAIDS/WHO (2004). Curable sexually transmitted infections UNAIDS/WHO (2004) Epidemiological Fact Sheet - 2004 Update $\mathrm{pp} 7$

[35]. Wallace, C. (2009) Hidden HIV Reservoirs. CNRS Magazine

[36]. Weiss, H. , Dickson, K. E, Agot, K., et al. (2010) Male circumcision for HIV prevention: Current research and programmatic issue AIDS 24: $61-69$.

[37]. WHO (2010) Antiretroviral therapy for HIV infection in adults and adolescents: Recommendations for a Public Health Approach. 2010 Revision. WHO Library Cataloguing-in-Publication Data

[38]. Williams, B.G., Lloyd-Smith, J.O., Gouws, E., et al., (2006). The potential impact of male circumcision on HIV in Sub-Saharan Africa. PLoS Med Abstract: http: //amedeo.com /lit $\quad$ id = 16822094.

[39]. Yasushiro, et al. (2008) Shooting for HIV's weakness. E - Lab Biotechnology. http//www.e - Lab. org 